\title{
Diagnosis and Management of Laryngo-Pharyngeal Reflux
}

\author{
Khurshid H. Alam • Petros V. Vlastarakos
}

Received: 17 October 2011/Accepted: 4 April 2012/Published online: 22 August 2012

(C) Association of Otolaryngologists of India 2012

\begin{abstract}
The aim of the present paper was to analyze laryngo-pharyngeal reflux (LPR) manifestations in ENT patients, and present a diagnostic and treatment algorithm for appropriate management. Retrospective chart review of 150 patients, who had initially presented with symptoms suggestive of LPR. Treatment included the administration of omeprazole $20 \mathrm{mg}$ twice daily for at least 4 weeks. Unresponsive patients were also given metoclopramide $10 \mathrm{mg}$ twice daily for four additional weeks, and the dose of omeprazole was increased to $40 \mathrm{mg}$ twice daily, if they did not achieve complete symptom resolution. Only patients who became completely asymptomatic after LPR treatment were included in the study. As many as $18 \%$ of patients attending an ENT outpatient department benefited from anti-reflux treatment. A need to clear the throat represented the most common symptom, whereas interarytenoid oedema/congestion was the most common finding on flexible naso-endoscopy (62.67 and $72.7 \%$ of patients, respectively). The vast majority of patients responded after 4 weeks of treatment with omeprazole, however, as many as $32 \%$ of LPR patients achieved complete symptom control after the initial 4 week trial period. LPR seems to
\end{abstract}

\footnotetext{
K. H. Alam

ENT Department, Doctors Hospital and Medical Centre,

Johar Town, Pakistan

P. V. Vlastarakos

ENT Department, Lister Hospital, Coreys Mill Lane, Stevenage, Hertfordshire SG1 4AB, UK

P. V. Vlastarakos $(\square)$

33 Wetherby Close, Stevenage, Hertfordshire SG1 5RX, UK

e-mail: pevlast@hotmail.com; pevlast@yahoo.gr
}

be more common than previously reported in the literature. Appropriate management of LPR can prevent the symptomatic use of various medical treatments for related manifestations, and even surgical interventions, thus decreasing the overall patient morbidity.

Keywords Reflux · Gastro-esophageal · Laryngeal · Treatment $\cdot$ Hoarseness · Proton pump inhibitors

\section{Introduction}

Laryngo-pharyngeal reflux (LPR) represents a controversial subject for both diagnosis and treatment. A number of studies have shown that as much as $10 \%$ of the patients presenting to ENT outpatient clinics may suffer from LPR $[1,2]$. These patients may complain of a broad spectrum of symptoms including dry cough, the need of constantly clearing their throat, post-nasal discharge, foreign body sensation, voice changes etc.

Most of these patients have received repeated courses of antibiotics, or have been treated for asthma, sinusitis or chronic tonsillitis. Many patients had surgery (i.e. tonsillectomy, bilateral middle meatal antrostomies, reduction of the inferior turbinates), and a significant number has come to a point of giving up on orthodox medicine, and considering alternative treatments (i.e. homeopathic).

Koafman [3] defined the otolaryngologic manifestations of gastro-esophageal reflux in 1991, and LPR has since been accepted as different entity from gastro-esophageal reflux disease, although controversies regarding diagnosis and management still exist.

The aim of the present paper is to analyze LPR manifestations in ENT patients, and present a diagnostic and treatment algorithm for appropriate patient management. 


\section{Materials and Methods}

A retrospective chart review was performed in 150 patients, who had initially presented with symptoms suggestive of LPR. These symptoms included the need to clear the throat, globus sensation in the throat, dry cough, postnasal discharge, soreness or irritation in the area of the larynx, and choking attacks. Only patients who became completely asymptomatic after LPR treatment were included in the study.
The authors employed a reflux symptom index (RSI), which uses a 0-5 scale to describe the effect of the disease on patients' quality of life with regard to nine main symptom categories, and a reflux finding score (RFS), which quantifies the effect of the disease on eight areas of the larynx, as seen during flexible naso-endoscopy (Tables 1,2) [4], during the initial assessment of patients.

Criteria for starting LPR treatment were (a) RSI more than 10 [5], (b) RFS more than 7 [4], (c) no other identifiable cause of symptoms after detailed clinical

Table 1 Reflux symptom index [4]

Within the last month, how did the following problem affect you? 0 - No problem 5 - Severe problem

1. Hoarseness or a problem with your voice

2. Clearing your throat

3. Excess throat mucus or post nasal drip

4. Difficulty swallowing food, liquids or pills

5. Coughing after you ate or after lying down

6. Breathing difficulties and choking episodes

7. Troublesome or annoying cough

8. Sensation of something in your throat

9. Heart burn, chest pain, indigestion or stomach acid coming up

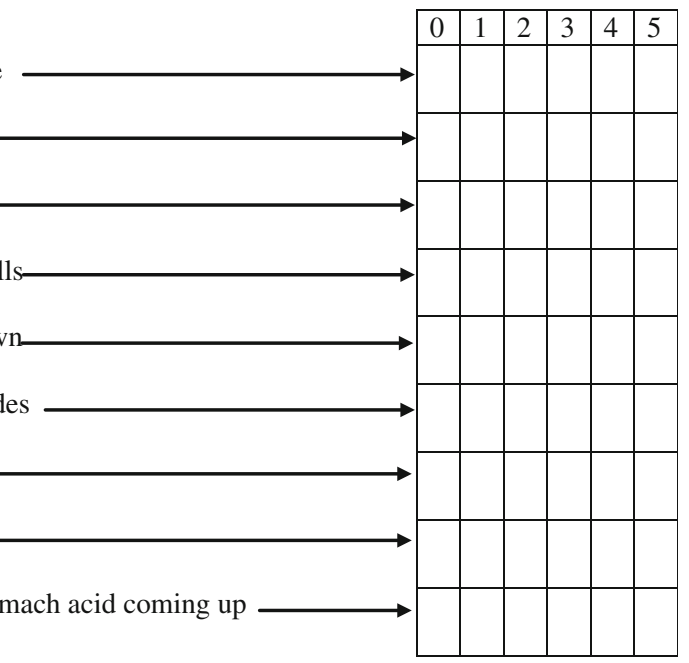

Table 2 Components of the reflux finding score [4]

1. Sub Glottic edema
Present $=2$
Absent $=0$

2. Ventricular obliteration

Partial $=2 \quad$ Complete $=4$

3. Erythema / hyperemia Arytenoids only $=2 \quad$ Diffuse $=4$

4. Vocal cord edema Mild $=1$

Polypoid $=4$

5. Diffuse laryngeal edema Mild $=1$

Moderate $=2$

Severe $=3$

6. Posterior commissure hypertrophy Mild $=1 \quad$ Moderate $=2$

Severe $=3 \quad$ Obstructing $=4$

7. Granuloma / granulation Present $=2 \quad$ Absent $=0$

8. Thick endolaryngeal mucus / others Present $=2 \quad$ Absent $=0$ 
examination, and related imaging, where appropriate (i.e. chest X-ray, CT scan of the sinuses, barium swallow etc.).

Patients were initially treated with a proton pump inhibitor (omeprazole) at the dose of $20 \mathrm{mg}$ twice daily and were reviewed after 4 weeks. If patients were not responsive during the 4 week trial period, metoclopramide was added to the treatment regimen in the dose of $10 \mathrm{mg}$ twice daily for 4 additional weeks. If the patients did not achieve complete symptom resolution, the dose of omeprazole was increased to $40 \mathrm{mg}$ twice daily for four more weeks.

\section{Results}

The study population represented $18 \%$ of patients who attended an ENT outpatient department within a time period of 2 years.

The mean age of patients presenting with LPR symptoms was 39.6 years (range $12-80$ years). The male to female ratio was equal. A need to clear the throat represented the most common symptom and was described by 94 patients $(62.67 \%)$. Other symptoms in descending frequency included dry cough (56\%), irritation or soreness in the area of the larynx $(53.33 \%)$, post-nasal discharge (42\%), husky voice $(41.33 \%)$, foreign body or globus sensation $(30.7 \%)$, nasal blockage $(28.7 \%)$, heartburn/ indigestion $(25.3 \%)$, and choking attacks $(17.3 \%)$.

Interarytenoid oedema/congestion was the most common finding on flexible naso-endoscopy and was seen in 109 patients $(72.7 \%)$. Other findings included interarytenoid band $(18.7 \%)$, vocal cord nodule/granuloma (5.3\%), and vocal cord oedema (3.3\%).

The vast majority of patients $(n=101)$ responded after 4 weeks of treatment with omeprazole given orally at the dose $20 \mathrm{mg}$ twice daily, whereas $22 \%$ required treatment for up to 8 weeks $(n=34)$, and $10 \%(n=15)$ required treatment for up to 12 weeks after their first visit to the outpatient department (Fig. 1).

\section{Discussion}

LPR was initially suggested as a term for the association of laryngeal disorders and gastro-esophageal reflux disease (GERD). Soon after this association was noticed, however, LPR was defined as being a different disease from GERD. Indeed, LPR tends to occur during the daytime in the upright position, and is not associated with obesity [5]. In addition, it presents with usually normal oesophageal motility, and most patients do not have oesophagitis as is always the case in GERD [6]. Nevertheless, different research groups have proposed different theories for the pathogenesis of LPR, considering it either as a retrograde

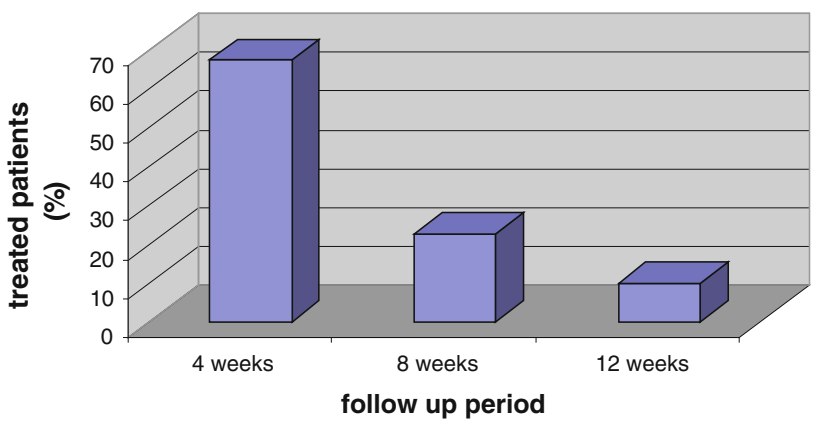

Fig. 1 Longitudinal patient response to LPR treatment

flow of gastric contents to the laryngopharynx [2], or an abnormal presence of gastric contents above level of the upper oesophageal sphincter [7]. Heterotrophic gastric mucosa in the cervical oesophagus could also play a role in the pathogenesis of LPR, at least in the minor group of patients with laryngitis of the posterior larynx [8].

Both LPR and GERD are caused by mucosal injury from acid and pepsin exposure, but the laryngeal epithelium is far more susceptible to reflux related tissue injury, than its oesophageal counterpart. In addition the oesophagus has an intrinsic antireflux defense system that prevents mucosal injury (bicarbonate production, mucosal tissue resistance and oesophageal motor function [9]), which in contrast seems to be less successful in the laryngo-pharynx.

The laryngeal epithelium does, however, express a limited amount of carbonic anhydrase, which can catalyze the hydration of carbon dioxide $\left(\mathrm{CO}_{2}\right)$ to produce bicarbonate $\left(\mathrm{HCO}_{3}{ }^{-}\right)$, and can hence protect itself against LPR to some extent. Moreover the $\mathrm{H}^{+} / \mathrm{K}^{+}$ATPase (proton) pump associated with the parietal cells of the stomach is present with some variable expression in the seromucinous cells and ducts of the human larynx. [10].

When the protective mechanisms fail, ciliary dysfunction and mucus stasis can occur. This can in turn cause accumulation of mucus, and produce a sensation post-nasal drip, which may induce throat clearing. Coughing and choking (laryngospasm) [7] can be induced by direct refluxate irritation, because the sensitivity in laryngeal sensory endings is up regulated by local inflammation [11, 12].

LPR seems to be more common than previously reported in the literature [2], as the present study identified that $18 \%$ of patients attending an ENT outpatient department benefited from anti-reflux treatment. The male to female patient ratio was, however, equal, in keeping with previous reports [13].

A frequent challenge when treating LPR is to convince the patients that the problem stems from the acid reflux. This task can be difficult, since $75 \%$ of the patients according to the results of the present study do not suffer from heartburn or any other common GERD-related symptoms. It should be noted that LPR has quite frequently been 
differentiated from GERD in that it is not associated with heartburn and other GERD symptoms. However, it appears that up to $25 \%$ of patients with LPR may present with various GERD-associated symptoms.

A 4 week trial of proton-pump inhibitors is frequently employed to confirm the clinical suspicion of LPR, as other diagnostic modalities (i.e. hypopharyngeal $\mathrm{pH}$ monitoring, multichannel intraluminal impedance and pH-metry [1417]), although more accurate especially in the presence of GERD symptoms, can be invasive, costly, associated with patient's discomfort, and not easily applied in clinical practice. Suspected patients initially undergo a complete ENT physical examination, and also chest auscultation, and $\mathrm{X}$-ray, if lung pathology is suspected. If the patients present with RSI score of more than 10, and/or RFS of more than 7 they are started on medical treatment and reviewed after 4 weeks (Fig. 2). Advice regarding life-style modifications (i.e. dietary changes, exercise, control of stress) are also given.

If patients are not responsive during the 4 week trial period, we can consider increasing the dose of omeprazole

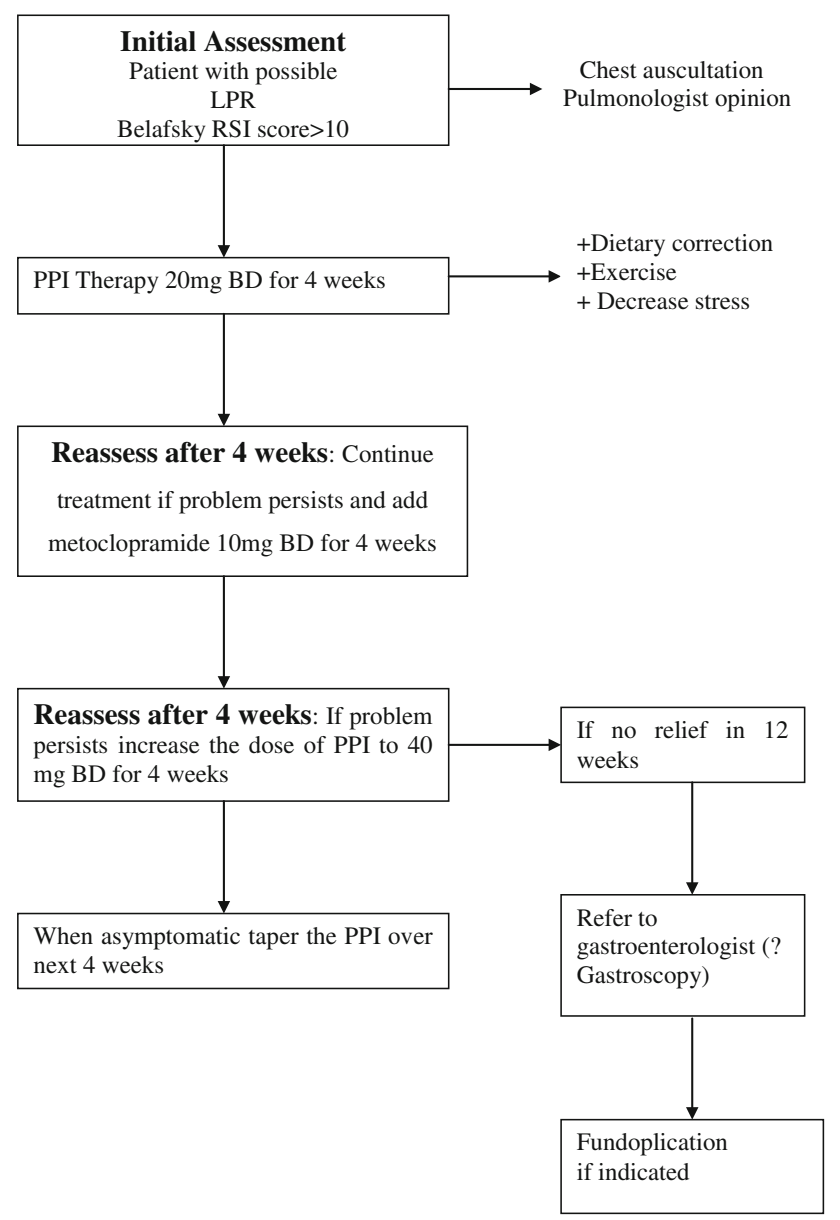

Fig. 2 Management algorithm for LPR patients up to $40 \mathrm{mg}$ twice daily $[7,18]$, or add metoclopramide to the treatment regimen. In the dose of $10 \mathrm{mg}$ twice daily, metoclopramide increases the tone of the gastro-esophageal junction [7], and can be prescribed for as long as the proton pump inhibitors are given. Co-administration of $\mathrm{H}_{2}$ inhibitors with proton pump inhibitors twice daily were also shown superior to daily proton pump inhibitors only [7].

A follow up period of up to 3 months is often required, because as many as $32 \%$ of LPR patients achieve complete symptom control after the initial 4 week trial period (Fig. 1). Finally, patients who are not responsive to treatment within the 3 month time period should be referred to the gastroenterologists for further management.

\section{Conclusion}

LPR seems to be more common than previously reported in the literature, and may be present in $18 \%$ of patients attending ENT outpatient clinics. A 4 week trial of protonpump inhibitors can be easily used to confirm the clinical suspicion of LPR, however, a follow up period of up to 3 months is often required, as a significant proportion of LPR patients achieve complete symptom control after the initial 4 week trial period. Appropriate management of LPR can prevent the symptomatic use of various medical treatments for related manifestations, and even surgical interventions, thus decreasing the overall patient morbidity.

Conflict of interest None.

\section{References}

1. Dale OT, Alhamarneh O, Young K, Mohan S (2010) Laryngeal sensory testing in the assessment of patients with laryngopharyngeal reflux. J Laryngol Otol 124:330-332

2. Bove MJ, Rosen C (2006) Diagnosis and management of laryngopharyngeal reflux disease. Curr Opin Otolaryngol Head Neck Surg 14:116-123

3. Koafman JA (1991) The otolaryngologic manifestations of GE R D: a clinical investigation of 225 patients using ambulatory 24 hours $\mathrm{pH}$ monitoring and an experimental investigation of the role of acid and pepsin in the development of laryngeal injury. Laryngoscope 101(Suppl 53): 1-78

4. Belafsky PC, Postma GN, Koafman A (2002) Validity and reliability of the reflux symptoms index (RSI). Voice 16:274-277

5. Celik M, Ercan I (2006) Diagnosis and management of laryngopharyngeal reflux disease. Curr Opin Otolaryngol Head Neck Surg 14:150-155

6. Remacle M (2006) Diagnosis and management of LPRD. Curr Opin Otolaryngol Head Neck Surg 14:143-149

7. Divi V, Bennirges MS (2006) Diagnosis and management of LPRD. Curr Opin Otolaryngol Head Neck Surg 14:124-127

8. Akbayi N, Sokmen HM, Callis AB et al (2005) Heterotropic gastric mucosa in the cervical esophagus: could this play a role in the pathogenesis of laryngopharyngeal reflux in a sub group of 
patients with posterior laryngitis? Scand J Gastroenterol 40: $1149-1156$

9. Johnston N, Knight J, Dettmar PW, Lively MO, Koufman J (2004) Pepsin and carbonic anhydrate isoenzyme III as diagnostic maskers for laryngopharyngeal reflux disease. Laryngoscope 114:2129-2213

10. Altman KW, Waltonen JD, Hammer ND, Radosevich JA, Haines GK 3rd (2005) Proton pump (H+/K+ ATPase) expression in human laryngeal seromucinous glands. Otolaryngol Head Neck Surg 133:718-724

11. Pontes P, Tiago R (2006) Diagnosis and management of LPRD. Curr Opin Otolaryngol Head Neck Surg 14:138-142

12. Aviv JE, Liu H, Parides M et al (2000) Laryngopharyngeal sensory deficits in patients with laryngopharyngeal reflux and dysphagia. Ann. Otol Rhino Laryngol 109:1000-1006

13. Moshkowitz M, Horowitz N, Halpern Z, Santo E (2011) Gastroesophageal reflux disease symptoms: prevalence, sociodemographics and treatment patterns in the adult Israeli population. World J Gastroenterol 17(10):1332-1335

14. Noordzij JP, Khidr A, Desper E et al (2002) Correlation of $\mathrm{pH}$ probe-measured laryngopharyngeal reflux with symptoms and signs of reflux laryngitis. Laryngoscope 112:2192-2195

15. Bardan E (2003) Pharyngoesophageal pH monitoring. Am J Med 115(Suppl 3A):78S-80S

16. Dobhan R, Castell DO (1993) Normal and abnormal proximal esophageal acid exposure: results of ambulatory dual-probe $\mathrm{pH}$ monitoring. Am J Gastroenterol 88:25-29

17. Vaezi MF, Schroeder PL, Richter JE (1997) Reproducibility of proximal probe $\mathrm{pH}$ parameters in 24 hours ambulatory esophageal pH monitoring. Am J Gastroenterol 92:825-829

18. Mahien HF, Frity Smith C (2006) Diagnosis and management of LPRD. Curr Opin Otolaryngol Head Neck Surg 14:133-137 